\section{Functional outcome of subthreshold versus threshold diode laser photocoagulation in diabetic macular oedema}

V Kumar, B Ghosh, DK Mehta and N Goel

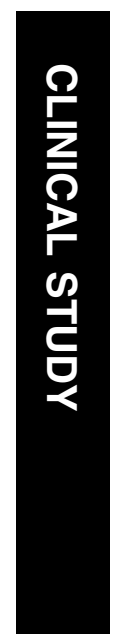

(on OCT) and in improving BCVA. Recovery of macular function as assessed by CS and PSRT maybe better with subthreshold laser. Eye (2010) 24, 1459-1465; doi:10.1038/eye.2010.53; published online 30 April 2010

Keywords: diabetic macular oedema; diode laser; subthreshold; contrast sensitivity; photo stress recovery time

\section{Introduction}

Macular oedema is leading cause of visual loss in diabetic patients. ${ }^{1}$ The Early Treatment of Diabetic Retinopathy Study (ETDRS) stated that prompt focal or grid laser photocoagulation for clinically significant macular oedema (CSME) reduced the incidence of moderate visual loss (doubling of the visual angle) by $50 \%$ at the end of 3 years. $^{2}$ Though the ETDRS used argon blue-green or argon green laser $(514 \mathrm{~nm})$, efficacy of krypton red $^{3}(647 \mathrm{~nm})$ and diode ${ }^{4}$ $(810 \mathrm{~nm})$ has been shown in modified grid laser for diffuse diabetic macular oedema (DDME).

The thermal effects of laser can however lead to complications, ${ }^{5}$ such as transient blurred vision, colour vision defects and loss of hue discrimination, paracentral scotomas, occurrence of choroidal neovascular membranes, and late enlargement of laser scars. ${ }^{6}$ As these side effects are due to collateral damage to the neurosensory retina while using visible end point of photocoagulation, they can be avoided by using a subvisible end point of laser and imparting laser energy selectively to the retinal pigment epithelium (RPE), the main site of action of laser. ${ }^{7}$
Guru Nanak Eye Centre, Maulana Azad Medical College, New Delhi, India

Correspondence: N Goel, Guru Nanak Eye Centre, Maulana Azad Medical College, Road No. 9, House No. 2, Punjabi Bagh Extension, New Delhi 110026, India Tel: + 919811179191 ; Fax: +911123230033. E-mail: nehadoc@hotmail. com

Received: 22 November 2009

Accepted in revised form: 10 March 2010 Published online: 30 April 2010 
Olk and Akduman ${ }^{8}$ performed a pilot study to determine the efficacy of subthreshold diode modified grid laser for DDME. Subsequently, few studies have been carried out on subthreshold laser and they have shown the anatomical resolution of oedema and improvement in visual acuity to be comparable to threshold laser. ${ }^{9}$ However, the functional outcome of either modality has not been compared. We conducted this prospective, comparative interventional case series between February and December 2008 to study the effect of subthreshold diode laser photocoagulation and compare it with conventional threshold laser in terms of change in best corrected Snellen visual acuity (BCVA), central macular thickness (CMT) on optical coherence tomography (OCT), photo stress recovery time (PSRT), contrast sensitivity (CS), and central visual field (VF).

\section{Materials and methods}

Thirty eyes of 20 patients were recruited from the Retina Clinic, Guru Nanak Eye Centre, New Delhi. The study was approved by the institutional review board and was performed in accordance with the tenets of the Declaration of Helsinki. Informed consent was obtained from all patients at the outset.

We included Type 1 and Type 2 diabetics aged 18 years or older with CSME detected on slit lamp biomicroscopy according to ETDRS criteria, ${ }^{2}$ leakage confirmed by intravenous fundus fluorescein angiography (FFA; VISUPAC and FF450 plus, Carl Zeiss Meditec Inc., Dublin, CA, USA), CMT $\geqslant 250 \mu \mathrm{m}$ on OCT (Stratus OCT, Carl Zeiss Meditec Inc.), and BCVA of 20/80 or better.

To gauge the systemic factors that might affect the severity of diabetic macular oedema (DME), all patients underwent blood pressure (BP) measurements and blood tests for glycosylated haemoglobin (Hba1c), renal function, and fasting lipid profile at baseline. Patients with diastolic $\mathrm{BP}>100 \mathrm{~mm} \mathrm{Hg}, \mathrm{Hba1c} \geqslant 8 \%$, or patients in renal failure requiring any type of dialysis were excluded.

Ocular exclusion criteria included macular ischemia $\geqslant 6$ clock hours on FFA, vitreo macular traction on OCT, proliferative diabetic retinopathy (PDR), previous laser photocoagulation for DME or history of intravitreal drug injection, and significant media opacities or any intra-ocular surgery in the preceding 12 months.

Baseline examination included BCVA, slit lamp examination, fundus biomicroscopy with a volk area centralis contact lens (Volk Corporation, Mentor, $\mathrm{OH}$, USA), FFA, and OCT. A fast macular protocol was used to assess the macular thickness and macular volume. It comprises six radial $6 \mathrm{~mm}$-long scans of each eye centred on the patient's fixation point, oriented at intervals of $30^{\circ}$. This mapping averaged the six scans to give the CMT in a central area $1 \mathrm{~mm}$ in diameter. In addition, the macular function was assessed with PSRT, CS, and 10-2 VF testing.

\section{Photo stress recovery time}

With undilated pupils and the other eye covered, the patient looked directly into the light of a handheld ophthalmoscope held about $3 \mathrm{~cm}$ away from the eye to be tested for a period of $10 \mathrm{~s}$. Immediately after the light was removed, the patient was asked to start reading Snellen test letters one line larger than the BCVA. The time (in seconds) required to read any three of the test letters just larger than the BCVA (ie, PSRT) was recorded. To ensure consistent 'dazzle', an ophthalmoscope that was dedicated to the study and fully charged was used. A recovery time of less than $50 \mathrm{~s}$ constitutes a normal reaction.

\section{Contrast sensitivity}

Pelli Robson chart, commercially available from Clement Clarke International Ltd., London (1988) was used to measure CS. ${ }^{11}$ It has eight lines of Sloan letters, each subtending an angle of $3^{\circ}$ at a test distance of $1 \mathrm{~m}$. The letters are arranged in triplets, each set progressively decreases in contrast by 0.15 log units, from 0.00 to 2.25 $\log$ units. Each line contains two triplets. The chart is wall mounted (measuring $60 \mathrm{~cm} \times 85 \mathrm{~cm}$ ) with a luminance of $90 \mathrm{~cd} / \mathrm{m}^{2}$. The test was done with an undilated pupil, keeping the other eye covered. When the patient made two mistakes within one triplet, the test was terminated and scored by letter.

\section{0-2 VF testing}

All patients were submitted to standard automated perimetry using 10-2 VF threshold testing with a size III stimulus (Humphrey Field Analyser II, Humphrey Instruments Inc., San Leandro, CA, USA) in the manner described in the User's guide. ${ }^{12}$ We designated VF 10-2 results as abnormal if they met moderately strict grading criteria for $30^{\circ}$ glaucomatous field loss, ${ }^{13}$ because grading criteria do not exist for diabetic retinopathy. These criteria include $\geqslant 3$ adjacent points of $\geqslant 5 \mathrm{~dB}$ loss each or $\geqslant 2$ adjacent points of $\geqslant 10 \mathrm{~dB}$ loss each. Field loss may be relative to normal values or values of surrounding points. The results were considered poorly reliable if false-positive or false-negative responses exceeded $33 \%$ or fixation losses exceeded $20 \% .^{13}$

Eligible eyes were randomised using a centralised computer-generated randomisation list with fixed permuted block for each site. Each patient was assigned to receive subthreshold (group A) or threshold (group B) 
diode laser photocoagulation within 1 week of baseline. The treating ophthalmologist was not masked to the treatment group because of differences in the technique of laser photocoagulation but the patients and investigators performing measurement of BCVA, PSRT, CS, OCT, and 10-2 VF were unaware of the allocated group.

Treatment was performed as an outpatient procedure under topical anaesthesia and mydriasis by a single surgeon (BG). A slit lamp integrated diode photocoagulator using $810 \mathrm{~nm}$ wavelength (Iris Medical OcuLight SLx, IRIDEX Corporation, Mountain Veiw, CA, USA) was used with ocular mainster (standard) focal/ grid contact lens (Ocular Instruments, Bellevue, WA, USA) applied to the cornea with methylcellulose fluid. A test spot was applied to the retina nasal to the optic disc using $200 \mathrm{~ms}$ duration, and the power was increased to produce a mild grey lesion (visible burn) at the level of the outer retina. For eyes assigned to group B, this intensity was applied in a modified grid pattern as described below. For eyes assigned to group A, the energy needed for the visible burn was kept constant but the duration was halved to $100 \mathrm{~ms}$ and treatment was carried out. ${ }^{8}$

Two or three rows of laser spots were applied in the para foveal region upto and including the edge of the foveal avascular zone. Spot size was $125 \mu \mathrm{m}$ in the para foveal area and spots were applied one spot size apart from each other. Then, $200 \mu \mathrm{m}$ spots were applied one burn width apart throughout all the remaining areas of retinal thickening and in all areas of capillary nonperfusion. Additional spots were applied focally in areas of obvious leakage.

Patients were scheduled for follow-up examination at weeks 1, 6, 12, and 18 after treatment. The BCVA, PSRT, CS, slit lamp examination, and fundus biomicroscopy were re-assessed at each of these visits. OCT was repeated at 6,12 , and 18 weeks whereas FFA and 10-2 VF were carried out at 12 and 18 weeks. All these parameters were tested by the same person, who was masked as to the patient's treatment status. All patients completed the desired follow-up at the end of 18 weeks. No re-treatments were carried out.

Outcome measures used to compare the two modalities of laser photocoagulation included anatomical effects (ie, change in CMT on OCT), functional effects through BCVA, PSRT, CS, and objective evidence of paracentral scotomas on 10-2 VF testing.

Patient data were recorded in data collection sheets. Statistical analysis was performed using Statistical Package for Social Sciences (SPSS) software version 10.1.0 (SPSS Inc., Chicago, IL, USA). Statistical differences between pre- and post-laser clinical data were assessed using Wilcoxon signed-rank test and the difference between subthreshold and threshold laser group (groups A and B, respectively) was calculated by Mann-Whitney $U$-test with $P<0.05$ considered significant. The data are presented as mean \pm SD.

\section{Results}

Thirty eyes of 20 patients (9 males and 11 females) with DME were recruited with 15 eyes in each group (Table 1 ). All patients were of Indian origin. The ages of the patients ranged from 40 to 62 years with a mean of $50.37 \pm 6.3$ years. All patients had type 2 diabetes controlled on diet and/or oral hypoglycaemic agents, and the duration of diabetes ranged from 7 to 14 years with a mean of $9.77 \pm 1.7$ years. The Hba1c averaged $7.08 \pm 0.5 \%$. Ten of $20(50 \%)$ patients had a history of hypertension and were controlled on oral antihypertensive drugs.

The duration of symptoms attributable to DME ranged from 16 to 36 weeks with a mean value of $24.6 \pm 0.6$ weeks. There was no history of any other ocular disease except for refractive errors or cataract. Five eyes in each group $(33 \%)$ were pseudophakic with a posterior chamber intra-ocular lens, remaining were phakic. Two eyes in group A and three in group B had early immature senile cataract. None of the eyes had progression of

Table 1 Baseline clinical characteristics of 20 patients with CSME

\begin{tabular}{|c|c|c|c|c|}
\hline & All eyes $(\mathrm{n}=30)$ & Group A $(\mathrm{n}=15)$ & Group B $(\mathrm{n}=15)$ & $\mathrm{P}-v a l u e^{\mathrm{a}}$ \\
\hline Age (years) & $50.37 \pm 6.3$ & $50.93 \pm 6.6$ & $49.8 \pm 6.2$ & 0.633 \\
\hline \multicolumn{5}{|l|}{ Gender } \\
\hline Male & 15 & 8 & 7 & 1 \\
\hline Female & 15 & 7 & 8 & 1 \\
\hline Duration of diabetes (years) & $9.77 \pm 1.7$ & $9.60 \pm 1.5$ & $9.93 \pm 1.9$ & 0.599 \\
\hline Duration of symptoms (weeks) & $24.6 \pm 6$ & $24.0 \pm 6$ & $25.2 \pm 6$ & 0.583 \\
\hline Hba1c $(\%)$ & $7.08 \pm 0.5$ & $7.11 \pm 0.5$ & $7.05 \pm 0.5$ & 0.730 \\
\hline
\end{tabular}

aStudent's $t$-test.

Values are mean \pm SD. 

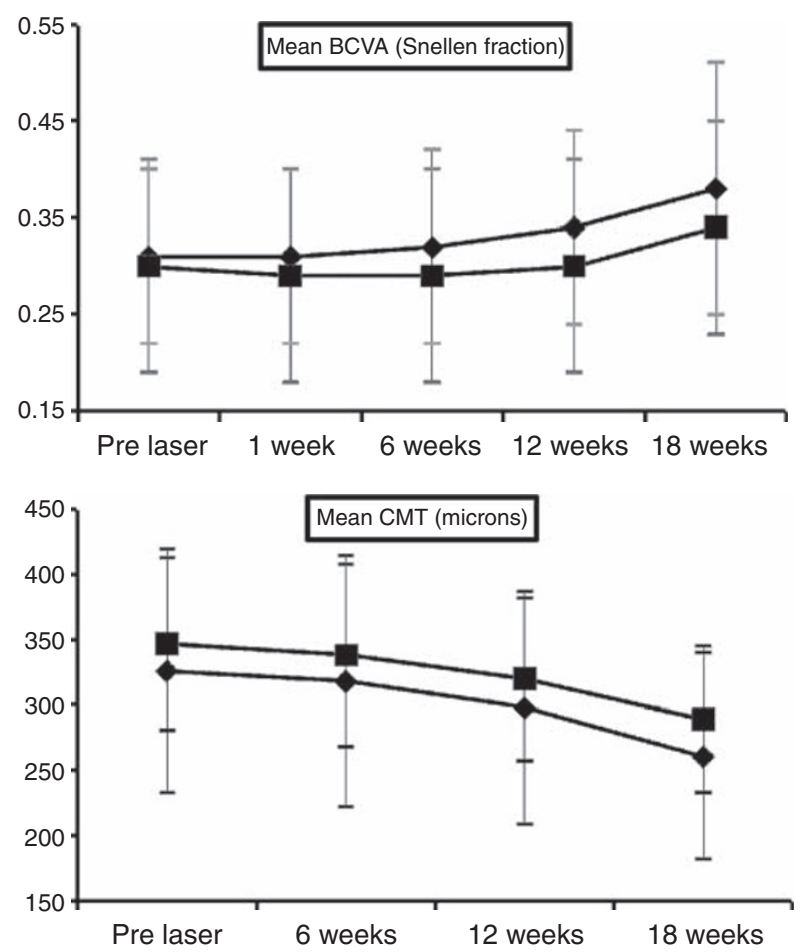

Figure 1 Comparison of the clinical course between the subthreshold diode laser-treated eyes (diamond) and threshold laser-treated eyes (square) in patients with clinically significant macular oedema (CSME). Top: comparison of the clinical course of best corrected Snellen visual acuity (BCVA). Each point and vertical bar indicates mean BCVA (Snellen fraction) \pm SD of the mean. Bottom: comparison of the clinical course of central macular thickness (CMT). Each point and vertical bar indicates mean CMT $(\mu \mathrm{m}) \pm \mathrm{SD}$ of the mean.

lenticular opacities during the follow-up. Six eyes in each group (40\%) had cystoid macular oedema (CME) on OCT, none of the eyes showed serous macular detachment. Of the 10 patients who had bilateral DME, each eye was randomly allocated to group A or B.

There were no significant differences between the two treatment groups with regard to baseline characteristics (Table 1).

Mean BCVA improved in group A after the first week. In group B, it decreases marginally till 6 weeks, after which it started to rise (Figure 1). For both the groups, the improvement in mean BCVA reached statistical significance $(P=0.03)$ only at 18 weeks visit (Table 2 ). Comparison between the two groups showed no significant difference at any visit. There were significant reductions $(P<0.01)$ in mean $\mathrm{CMT}$ at all visits compared with baseline in both the groups (Table 2). Comparisons of the two groups showed no significant difference at all visits.

In both the groups, PSRT showed a significant increase $(P<0.001)$ at first week (Table 2$)$. In group $A$, the value subsequently declined significantly $(P<0.001)$ at each
Table 2 Change in outcome measures after laser photocoagulation

\begin{tabular}{|c|c|c|c|c|c|}
\hline & Group A & P-value ${ }^{\mathrm{a}}$ & Group B & P-value & P-value \\
\hline \multicolumn{6}{|c|}{ Mean BCVA (Snellen fraction) } \\
\hline Pre-laser & $0.31 \pm 0.09$ & - & $0.30 \pm 0.11$ & - & 0.768 \\
\hline 1 week & $0.31 \pm 0.09$ & - & $0.29 \pm 0.11$ & 0.334 & 0.659 \\
\hline 6 weeks & $0.32 \pm 0.10$ & 0.334 & $0.29 \pm 0.11$ & 0.334 & 0.478 \\
\hline 12 weeks & $0.34 \pm 0.10$ & 0.112 & $0.30 \pm 0.11$ & 0.581 & 0.327 \\
\hline 18 weeks & $0.38 \pm 0.13$ & 0.032 & $0.34 \pm 0.11$ & 0.030 & 0.323 \\
\hline \multicolumn{6}{|l|}{ CMT $(\mu m)$} \\
\hline Pre-laser & $326 \pm 93$ & - & $347 \pm 66$ & - & 0.474 \\
\hline 6 weeks & $318 \pm 96$ & 0.007 & $338 \pm 70$ & 0.003 & 0.536 \\
\hline 12 weeks & $298 \pm 89$ & 0.000 & $320 \pm 63$ & 0.000 & 0.449 \\
\hline 18 weeks & $261 \pm 79$ & 0.000 & $289 \pm 56$ & 0.000 & 0.283 \\
\hline \multicolumn{6}{|l|}{ PSRT (s) } \\
\hline Pre-laser & $75 \pm 16$ & - & $81 \pm 12$ & - & 0.295 \\
\hline 1 week & $76 \pm 16$ & 0.000 & $84 \pm 11$ & 0.000 & 0.161 \\
\hline 6 weeks & $73 \pm 16$ & 0.000 & $82 \pm 11$ & 0.013 & 0.085 \\
\hline 12 weeks & $70 \pm 17$ & 0.000 & $80 \pm 11$ & 0.181 & 0.072 \\
\hline 18 weeks & $66 \pm 17$ & 0.000 & $78 \pm 11$ & 0.003 & 0.032 \\
\hline \multicolumn{6}{|c|}{ CS (log units) } \\
\hline Pre-laser & $0.72 \pm 0.3$ & - & $0.68 \pm 0.2$ & - & 0.693 \\
\hline 1 week & $0.71 \pm 0.3$ & 0.334 & $0.62 \pm 0.2$ & 0.054 & 0.316 \\
\hline 6 weeks & $0.78 \pm 0.3$ & 0.028 & $0.66 \pm 0.2$ & 0.498 & 0.232 \\
\hline 12 weeks & $0.93 \pm 0.3$ & 0.000 & $0.69 \pm 0.2$ & 0.774 & 0.028 \\
\hline 18 weeks & $1.04 \pm 0.3$ & 0.000 & $0.73 \pm 0.3$ & 0.387 & 0.013 \\
\hline
\end{tabular}

${ }^{a}$ Wilcoxon signed-rank test (comparison within the group).

${ }^{b}$ Mann-Whitney $U$-test (comparison between the two groups). Values are mean \pm SD.

Values in bold indicate significant $P$-value $(<0.05)$.

visit, whereas in group $B$, the decrease was significant at weeks 6 and $18(P<0.02)$. At the final observation visit, the PSRT in the subthreshold group was significantly better than that in the group receiving threshold treatment $(P=0.03)$, though it did not attain a normal value (Figure 2).

CS worsened slightly in both the groups at the first follow-up. After this, it improved significantly $(P<0.03)$ in group $A$, at each visit (Table 2). Though CS in group $B$ also increased steadily, this failed to achieve significant proportions at any time. Comparison between the two groups (Figure 2) showed that the CS in the subthreshold group was significantly better at 12 and 18 weeks of follow-up $(P<0.03)$.

One patient in group $B$ showed presence of a paracentral scotoma on 10-2 VF after the laser photocoagulation, which persisted till the end of follow-up. No change was noticed in the pre-existing scotomas (two patients in group A and one in group B).

Slit lamp biomicroscopy showed evidence of decrease in macular thickening, haemorrhages, and hard exudates in all eyes. Leakage resolved or decreased in all eyes except one in each group (notably, both these patients had CME on OCT). Laser spots were visible clinically in 

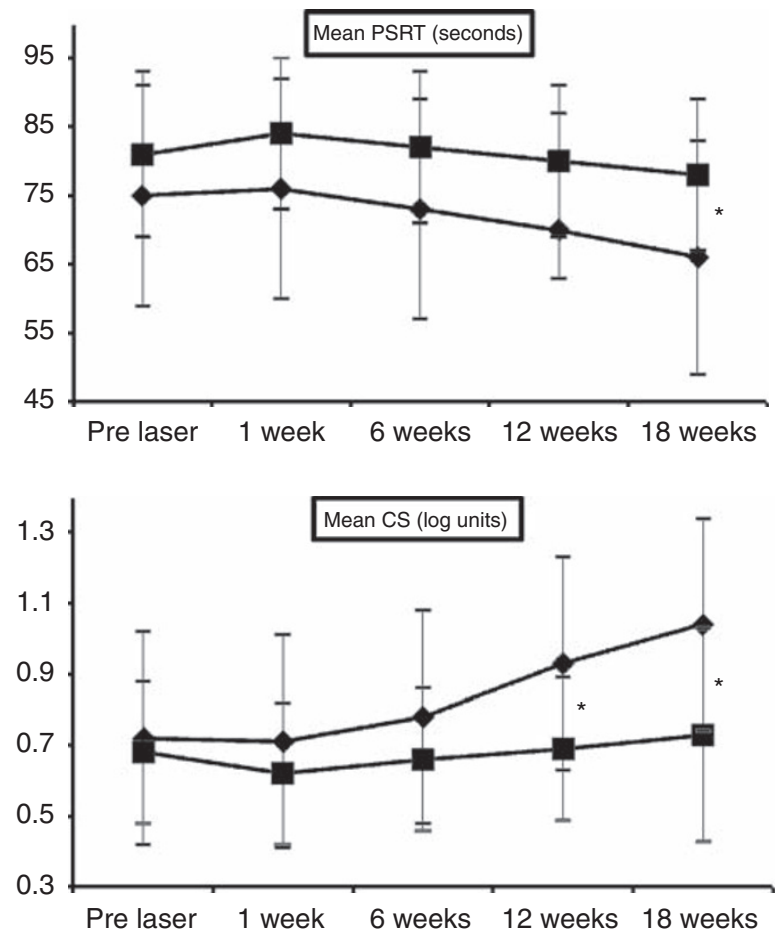

Figure 2 Comparison of the clinical course between the subthreshold diode laser-treated eyes (diamond) and threshold laser-treated eyes (square) in patients with clinically significant macular oedema (CSME). Top: comparison of the clinical course of photo stress recovery time (PSRT). Each point and vertical bar indicates mean PSRT (seconds) $\pm \mathrm{SD}$ of the mean. Bottom: comparison of the clinical course of contrast sensitivity (CS). Each point and vertical bar indicates mean CS (log units) \pm SD of the mean. The asterisk $\left(^{*}\right)$ indicates a statistically significant difference between the two groups at each time point $(P<0.05)$.

all eyes in group $\mathrm{B}$ and only in one eye in group $\mathrm{A}$. However, on FFA, all the eyes in both the groups had evidence of laser spots. None of the patients developed ischemic maculopathy or PDR during the period of observation. Complications such as choroidal neovascularisation, sub-retinal fibrosis, or inadvertent foveal photocoagulation did not occur in any patient of either group. Figure 3 shows a representative patient in group A (case 2).

\section{Discussion}

The value of conventional laser photocoagulation is well established for CSME in diabetics. ${ }^{2}$ A visible laser burn is applied to the retina following which laser light absorption heats pigmented tissue at the laser site. Heat conduction spreads this temperature increase from the RPE and choroid to the overlying non-pigmented and adjacent unexposed tissues. Threshold laser lesions thus become visible immediately because of a change in the scattering properties of the retina because of tissue coagulation of photoreceptors.

The RPE has an important function in the therapeutic effect of laser photocoagulation in DME. This suggests a benefit from targeting the RPE instead of the sensory retina. ${ }^{14}$ Energy deposition in the RPE restores normal transport mechanisms to and from the outer retinal layers, RPE, and choriocapillaris, which helps to resolve the retinal oedema. ${ }^{7,15}$ It has been hypothesised that RPE cells may spread and migrate at the edge of the lasered site or may divide by mitosis to explain this therapeutic effect. Cell division in retinal endothelium cells remote from the lasered site has also been observed suggesting that the mechanism maybe an indirect RPE-related effect. ${ }^{15}$

Collateral damage during laser photocoagulation can be reduced by using a longer wavelength laser. Although argon laser results in damage to both inner and outer retinal layers, diode laser near infrared radiation at $810 \mathrm{~nm}$ produces damage to the outer retina (including $\mathrm{RPE}$ ) and choroid only. The longer wavelength can penetrate more effectively through media opacities and thickened retina. Other well-known advantages of diode laser especially in context to its use in developing countries are its low cost, portability, lesser maintenance, and long operating life. ${ }^{16}$

Intrinsic damage from visible end point laser photocoagulation can also be decreased by reducing laser exposure duration and by using a subthreshold clinical end point for therapy. Subthreshold micropulse diode laser delivers energy as a series of very brief micropulses within a single laser exposure (envelope) thus limiting the rise in retinal temperature and has shown beneficial clinical results. ${ }^{17,18}$ Subthreshold (RPE) photocoagulation using diode laser and invisible end point of treatment has also been shown to be effective in reduction or elimination of DME, stabilisation, and improvement in BCVA, reduction of post-treatment paracentral scotomas both objectively and subjectively and resolution of posttreatment atrophic scarring. ${ }^{8,9}$

Most previous studies that have documented the efficacy of laser photocoagulation in preserving visual function in DME have used visual acuity as the sole parameter. Although visual acuity is a measure of the ability to resolve fine spatial details and is determined by using high contrast letters, CS is a measure of visual function in conditions of reduced contrast. CS maybe affected in patients with a Snellen visual acuity of $6 / 6$, hence its assessment is important for a more complete analysis of visual performance. ${ }^{19}$

PSRT has been used as a quantitative measure of macular function. ${ }^{10}$ The photostress response has been explained as a transient state of visual insensitivity caused by bleaching of the visual pigments. Return of 

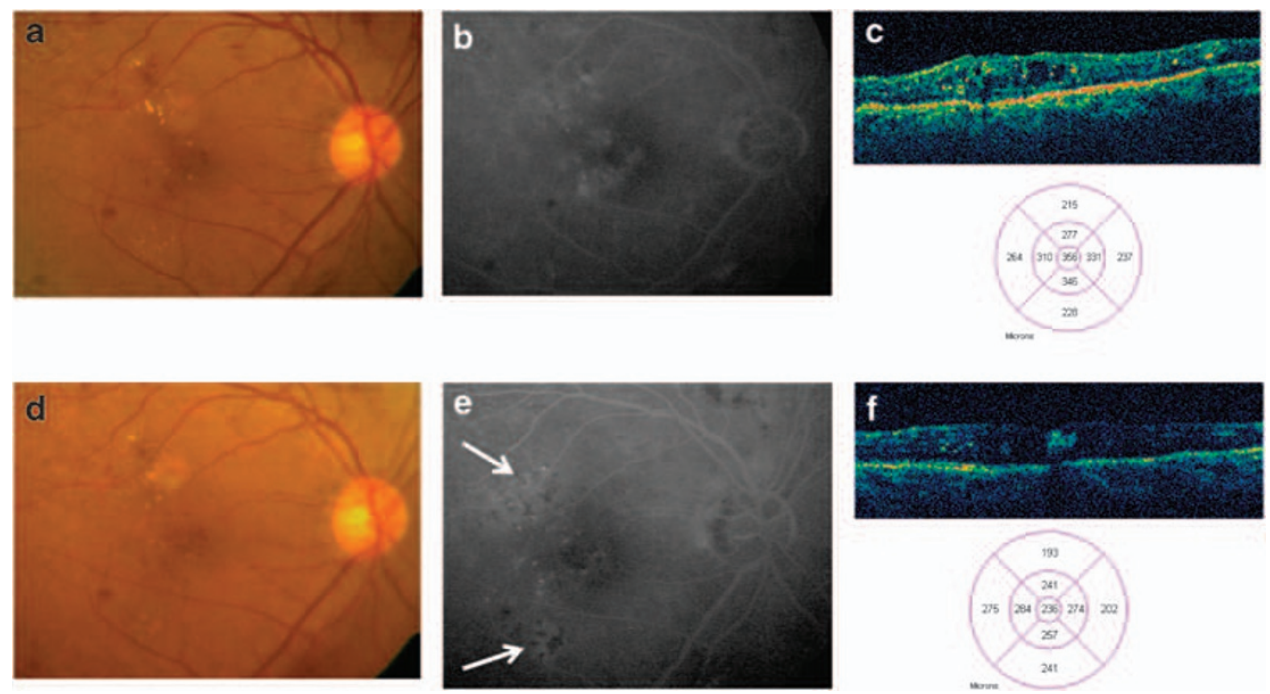

Figure 3 Case 2 (group A) - a 56-year-old female with history of diabetes since 9 years complained of decreased vision in her right eye since 12 weeks. Her Hba1c was 6.8\%. Best-corrected visual acuity (BCVA) was 0.25 (Snellen fraction) in the right eye with clinically significant macular oedema (CSME) on fundus examination (a), macular leakage on fluorescein angiography (b), and macular thickness as shown (c). Contrast sensitivity (CS) and photo stress recovery time (PSRT) were 0.9 log units and $95 \mathrm{~s}$, respectively. She received subthreshold diode laser photocoagulation following which BCVA improved to 0.5, with resolution of oedema clinically (d), angiographically (e), and on OCT (f) by 12 weeks. CS and PSRT improved to $0.45 \mathrm{log}$ units and $56 \mathrm{~s}$, respectively. Laser spots are not visible clinically, but can be seen on angiography (arrows).

sensitivity is dependent on re-synthesis of the visual pigment - this requires adequate perfusion of the photoreceptors and RPE. Thus, increased PSRT may result from anatomical derangement in the macula or decreased perfusion of the photoreceptors, and it maybe a sensitive tool to show recovery of macular function after treatment.

Our study was a prospective randomised controlled trial designed specifically to determine the efficacy of subthreshold diode laser photocoagulation in 30 eyes of 20 patients with DME and compared it with that of conventional laser in terms of anatomical (OCT) and functional outcome (BCVA, CS, PSRT, and central VF). In agreement with previously performed studies, ${ }^{8,9}$ we found that both treatment groups showed comparable efficacy in improving BCVA and reducing mean CMT on OCT when followed up for 18 weeks. CS and PSRT deteriorated in the first week, which may be due to the transient increase in macular oedema known to occur after laser treatment. However, significant improvement was attained in both parameters at an earlier point of time in the subthreshold group and values were significantly better in this group at the end of 18 weeks. This shows that recovery of macular function after laser photocoagulation maybe better and earlier with a subvisible clinical end point and maybe related to less damage to the neurosensory retina. The development of a paracentral scotoma in a patient receiving threshold treatment is of note, though this was not statistically significant.

Previous studies, which have compared subthreshold micropulse diode photocoagulation with conventional green laser, ${ }^{17}$ and 'light' and 'classic' Nd:YAG (frequency doubled) laser, ${ }^{20}$ showed comparable clinical results between the two modalities. Subthreshold diode laser and conventional threshold diode laser have not been compared in terms of effect on CS or PSRT.

The main problems of the subthreshold technique are related to dosimetry. Appropriate laser energy and necessary number of laser burns are unclear variables. Owing to the subtle nature of this method, lesions are only visible by FFA and not by ophthalmoscopy. Thus, there is no apparent end point to help titrate or confirm therapy. As tissue response with conventional photocoagulation is more pronounced because of larger magnitude of cellular response, it could be that more number of laser burns are necessary with subthreshold effects, though we did not statistically analyse this aspect. A case of choroidal infarct with large spot subthreshold infrared laser has been reported resulting in profound loss of vision immediately post laser. ${ }^{21}$

Independent of the potential problems of subthreshold laser, the clinical findings of our study show that it is not always necessary to produce retinal blanching and destroy photoreceptors to achieve a therapeutic effect. The energy used by the ETDRS in CSME may in fact be 
overtreating patients with its antecedent effects such as paracentral scotomas and post-treatment atrophic scarring. We conclude that subthreshold diode laser photocoagulation is effective in reducing or eliminating DME (as evidenced on OCT) and improving BCVA. Recovery of macular function as assessed by CS and PSRT maybe better and earlier as compared to threshold laser. This technique may avoid localised scotomas and allow re-treatment by minimising chorioretinal laser damage.

Our study is limited by its small sample size and short follow-up. As only a few small studies concerning the effect of subthreshold diode laser photocoagulation in DME have been performed so far, large controlled trials are needed to consider this as a routine treatment in DME.

\section{Summary}

What was known before

- Subthreshold diode laser photocoagulation is as efficacious as conventional threshold laser in improving visual acuity and reducing diabetic macular oedema. There is lesser collateral damage when subvisible end point of photocoagulatio is used.

What this study adds

- Subthreshold laser shows superior results as compared to threshold laser when contrast sensitivity and photo stress recovery time are used as parameters. Functional outcome with subthreshold laser maybe better than conventional laser, though visual acuity and anatomical resolution of oedema are comparable. Recovery of macular function maybe better and earlier with this modality.

\section{Conflict of interest}

The authors declare no conflict of interest.

\section{Acknowledgements}

This work has been presented in part as a poster at the 2008 Joint annual meeting of American Academy of Ophthalmology and European Society of Ophthalmology (Atlanta, USA). Funding/support: none.

\section{References}

1 Moss SE, Klein R, Klein BE. The 14-year incidence of visual loss in a diabetic population. Ophthalmology 1998; 105: 998-1003.

2 Early Treatment Diabetic Retinopathy Study Research Group. ETDRS Report no 1. Photocoagulation for diabetic macular edema. Arch Ophthalmol 1985; 103: 1796-1806.

3 Olk RJ. Argon green versus krypton red modified grid laser photocoagulation for diffuse diabetic macular edema. Ophthalmology 1990; 97: 1101-1113.
4 Akduman L, Olk RJ. Diode laser versus argon green modified grid photocoagulation for diffuse diabetic macular edema. Ophthalmology 1997; 104: 1433-1441.

5 Early Treatment Diabetic Retinopathy Study Group. ETDRS Report no 12. Fundus photographic risk factors for progression of diabetic retinopathy. Ophthalmology 1991; 98: 823-833.

6 Schatz H, Madeira D, McDonald HR, Johnson RN. Progressive enlargement of laser scars following grid laser photocoagulation for diffuse macular edema. Arch Ophthalmol 1991; 109: 1549-1551.

7 Bresnik GH. Diabetic maculopathy: a critical review highlighting macular edema. Ophthalmology 1983; 90: 1301-1317.

8 Akduman L, Olk RJ. Sub threshold (invisible) modified grid diode laser photocoagulation in diffuse diabetic macular edema. Ophthalmic Surg Lasers 1999; 30: 706-714.

9 Olk RJ, Akduman L. Minimal intensity diode laser (810 nanometer) photocoagulation (MIP) for diffuse diabetic macular edema (DDME). Semin Ophthalmol 2000; 16: $25-30$.

10 Glaser JS, Savino PJ, Sumers KD, McDonald SA, Knighton RW. The photo-stress recovery test in the clinical assessment of visual function. Am J Ophthalmol 1977; 83: 255-260.

11 Pelli DG, Robson JG, Wilkins AJ. The design of a new letter chart for measuring contrast sensitivity. Clin Vis Sci 1988; 2: 187-189.

12 Humphrey Instruments, Inc. Humphrey Field Analyzer II User's Guide. Humphrey Instruments Inc.: San Leandro, CA, 1994.

13 Caprioli J. Automated perimetry in glaucoma. In: Walsh TJ (ed). Visual Fields: Examination and Interpretation. American Academy of Ophthalmology: San Francisco, CA, 1990, pp 88.

14 Roider J. Laser treatment of retinal diseases by subthreshold laser effects. Semin Ophthalmol 1999; 14: 19-26.

15 Roider J, Brinkmann R, Wirbelauer C, Laqua H, Birngruber R. Subthreshold (retinal pigment epithelium) photocoagulation in macular diseases: a pilot study. $\mathrm{Br} \mathrm{J}$ Ophthalmol 2000; 84: 40-47.

16 Tewari HK, Gupta V, Kumar A, Verma L. Efficacy of diode laser for managing diabetic macular oedema. Acta Ophthalmol Scand 1998; 76: 363-366.

17 Figueira J, Khan J, Nunes S, Sivaprasad S, Rosa A, Faria de Abreu J et al. Prospective randomized controlled trial comparing subthreshold micropulse diode laser photocoagulation and conventional green laser for clinically significant diabetic macular oedema. Br J Ophthalmol 2008; 93(10): 1341-1344.

18 Laursen ML, Moeller F, Sander B, Sjoelie AK. Subthreshold micropulse diode laser treatment in diabetic macular ooedema. Br J Ophthalmol 2004; 88: 1173-1179.

19 Talwar D, Sharma N, Pai A, Azad RV, Kohli A, Virdi PS. Contrast sensitivity following focal laser photocoagulation in clinically significant macular oedema due to diabetic retinopathy. Clin Exp Ophthalmol 2001; 29: 17-21.

20 Bandello F, Polito A, Borrello MD, Zemella N, Isola M. 'Light' versus 'classic' laser treatment for clinically significant diabetic macular oedema. Br J Ophthalmol 2005; 89: 864-870.

21 Squirrell DM, Stewart AW, Joondeph BC, Danesh-Meyer $\mathrm{HV}$, McGhee CN, Donaldson ML. Large-spot subthreshold infrared laser to treat diabetic macular edema. Retina 2008; 28: 615-621. 